Original article

Central Eur J Paed 2018;14(1):81-87

DOI $10.5457 / \mathrm{p} 2005-114.203$

\title{
Clinical characteristics of pineal gland cysts in pediatric patients: A hospital based study
}

\author{
Matea Ćurković ${ }^{1}$, Ivana Unić Šabašov ${ }^{2}$ \\ ${ }^{1}$ University of Split, School of Medicine \\ Split, Croatia, ${ }^{2}$ Department of Pediatrics \\ University Hospital Split, Split, Croatia
}

Received: October 14, 2017

Accepted: January 3, 2018

Key words: Pineal gland cysts - Headache - Epilepsy - Central Nervous System Children.
Objective - The aim of this study was to analyze the clinical characteristics of patients under 18 years diagnosed with pineal gland cysts. Methods - The study was conducted on a sample of 79 children and adolescents diagnosed with pineal gland cysts found among the 30,150 children treated at our Department in a 10 -year period. We collected data for each child regarding gender, age, all pre-existing diagnoses, symptoms, all diagnostic methods performed in the patients and the type of treatment. Results - Out of 79 children, 47 had cysts whose size ranged from 0.51 to $1.0 \mathrm{~cm}$. The most common symptoms associated with the pineal gland cyst were headache $(21.5 \%)$ and general weakness $(12.6 \%)$. The most common diagnoses leading to pineal gland cyst diagnosis were epilepsy $(27.8 \%)$, change of psychological status (19\%), headache $(17.7 \%)$, tendency to atopy $(11.4 \%)$ and obesity (10.1\%). Most of the patients did not take drugs (44.3\%), whereas $29.1 \%$ were taking one drug as chronic therapy. The most commonly used drugs were anticonvulsants (27.3\%). Conclusion - Our study confirmed findings in adult patients with pineal gland cysts that headache and weaknesses, as well as diagnosis of epilepsy, are potentially related to pineal gland cyst diagnosis in children. Our conclusions may help accelerate the process of diagnosing these cysts and shorten the time of hospitalization, reduce the number of examinations and healthcare costs. Due to the rarity of this cyst, it would be desirable to repeat the research on a larger number of children.

\section{Introduction}

The pineal gland is placed behind the third ventricle in the central part of the brain, between the two hemispheres. It develops from the diencephalon roof plate (1). The pineal gland receives sympathetic, parasympathetic and sensory innervation. Sympathetic innervation is essential for secretion, while parasympathetic and sensory innervation are essential for feedback (2). It reaches its maximum size in the second year and retains this size for life (3).
The pineal gland cyst is a fluid-filled sac that is visible on 1.5 to $10.8 \%$ of magnetic resonance imaging brain scans, and $25-40 \%$ of autopsies (4). The exact cause of occurrence is unknown. Some studies have shown that bleeding in the pineal region and hormonal imbalance can enhance their development and growth. It has been widely accepted that cysts that are up to $10 \mathrm{~mm}$ in size are asymptomatic, whereas cysts whose size ranges from 7 to $45 \mathrm{~mm}$ and larger may be associated with symptoms. The association between 
the size of the cyst and the time of appearance of the first symptoms is not yet clear (5). There is not much information available about what happens to pineal gland cysts during a person's lifetime. It has been noted that most pineal cysts remain the same size throughout their lifetime and do not cause any symptoms, while a smaller amount of cysts decrease in size as patients grow older (6).

The symptoms pineal gland cysts may cause are headache, vertigo, visual and oculomotor disturbances, and obstructive hydrocephalus. Rarely, people with pineal cysts may have ataxia, motor and sensory impairment, mental and emotional disturbances, epilepsy, and circadian rhythm disturbances. The literature also describes cases of hormonal imbalance that may lead to premature puberty or secondary parkinsonism (5). Treatment of pineal gland cysts is diverse and depends on their size and associated symptoms. Asymptomatic patients do not require any treatment, but magnetic resonance imaging checkups are required, especially if the cysts are larger than $10 \mathrm{~mm}$. If the cyst does not grow, two MRI follow ups are enough (7). On the other hand, some studies have shown that routine MRI follow-up of pineal cysts is not necessary in the absence of unusual radiological characteristics or related clinical symptoms (11).

The objective of this study was to determine the clinical characteristics of children and adolescents diagnosed with pineal gland cysts. We analyzed the medical examinations needed to diagnose them, the medications the children received in long-term treatment, and the patients' psychological profile.

\section{Methods}

Retrospective analysis of data was conducted from medical documentation collected from the 30,150 children and adolescents treated at the Department of Pediatrics of the Clinical Hospital Center, Split in a 10year period from January 2006 to December 2016. Among these patients, we searched for children and adolescents diagnosed with pineal gland cysts. We collected data regarding gender, age, all pre-existing diagnoses, symptoms, all diagnostic methods performed in the patients and the type of treatment for each child separately.

Most children diagnosed with pineal gland cysts had numerous health issues and their psychological profile appeared to be similar, therefore we planned psychological testing to prove this. However, only six agreed to participate in the psychological testing so we decided not to proceed with it.

\section{Statistical analyses}

Symptoms and accompanying diagnoses were analyzed as descriptive statistics, due to the rarity of the observed diagnoses and small sample size. The amount of diagnostic procedures and drugs in chronic therapy were presented graphically using Microsoft Office Excell for Windows. To analyze the size of the pineal gland cysts, we used a Chi-Square test of MedCalc v. 11 for Windows (MedCalc Software, Mariakerke, Belgium). $\mathrm{P}<0.05$ was set as the statistical significance limit.

\section{Results}

The study included 79 children and adolescents, 31 (39\%) male and 48 (61\%) female. Most of the patients (59, 74.7\%) were hospitalized and $20(25.3 \%)$ received checkups in the Outpatient Clinic twice a year. Most of the children $(59.5 \%)$ had a cyst whose size ranged from $0.51 \mathrm{~cm}$ to $1.0 \mathrm{~cm}$, and the ChiSquare test proved statistical significance $\left(\chi^{2}\right.$ test $=40,265, \mathrm{df}=2, \mathrm{P}<0.001$ ) (Fig. 1).

The most common symptoms associated with the pineal gland cyst were headache $(21.5 \%)$, weakness $(12.6 \%)$ and loss of con- 


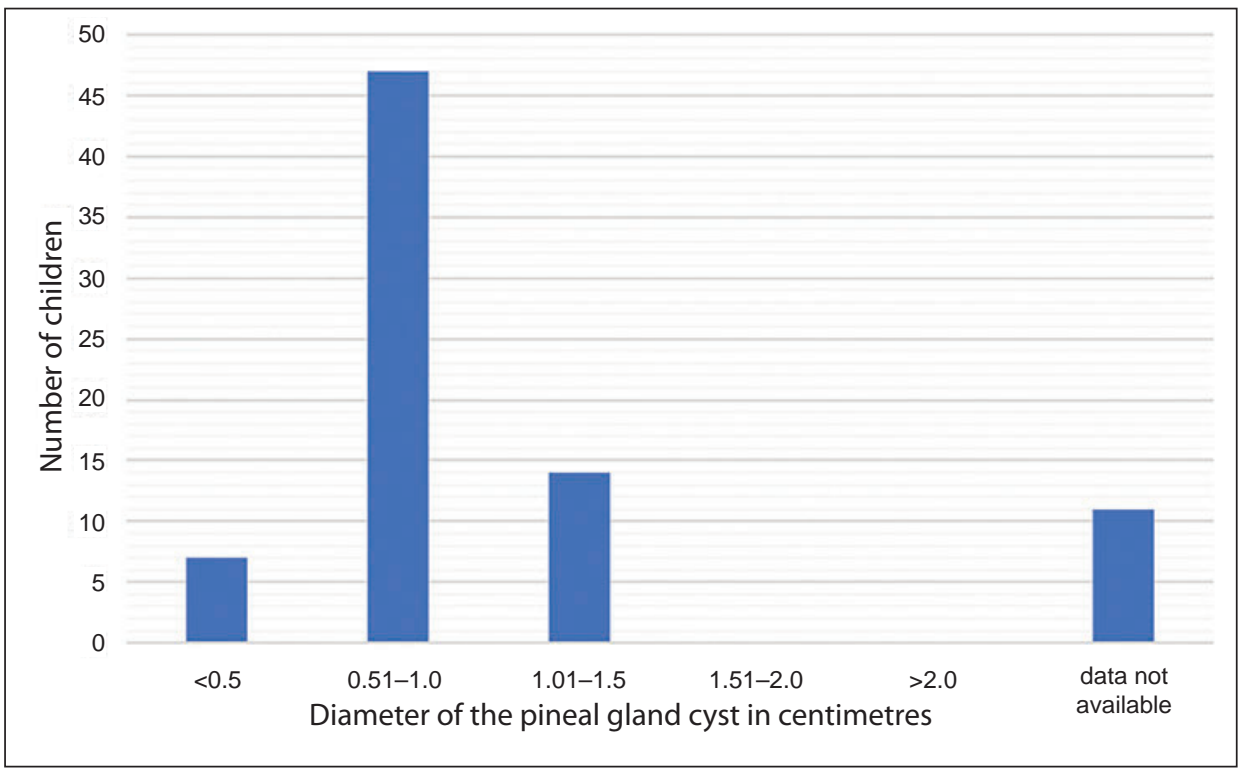

Fig. 1. Size of the pineal gland cyst in centimetres.

Table 1. Overview of all symptoms in the surveyed population of children*

\begin{tabular}{ll}
\hline Signs and symptoms & Presenting symptoms n; (\%) \\
\hline Nervous system + eye & $17(21.5)$ \\
\hline Headache & $10(12.6)$ \\
Weakness & $8(10.1)$ \\
Loss of consciousness & $7(8.8)$ \\
Vertigo & $5(6.3)$ \\
Blurred vision & $2(2.5)$ \\
Diplopia & $2(2.5)$ \\
Photophobia & \\
\hline Locomotor system & $6(7.6)$ \\
\hline Shivering & $3(3.8)$ \\
Stiffness in fingers & $3(3.8)$ \\
Tingling & $3(3.8)$ \\
Involuntary twitching of extremities & $2(2.5)$ \\
Knee pain & \\
\hline Digestive system & $3(3.8)$ \\
\hline Vomiting & $2(2.5)$ \\
\hline Nausea & $2(2.5)$ \\
\hline Endocrine system & \\
\hline Premature adrenarche & \\
\hline
\end{tabular}

*The symptoms presented in only one patient have not been included in the Table 1 (Hirsutism, dwarfism, tremor, menorrhagia, tingling, nervousness, sleepiness, forgetfulness). 
sciousness (10.1\%) (Table 1). The most common diagnoses leading to diagnosis of pineal gland cyst were epilepsy $(27.8 \%)$, change of psychological status (19\%), headache $(17.7 \%)$, tendency to atopy $(11.4 \%)$ and obesity (10.1\%). Change of psychological status encompasses various entities, such as: conversional disorders, psychosomatic disorders, somatization disorders, anxiety depressive disorder, attention deficit and hyperac-

Table 2. Overview of diagnoses in the children diagnosed with pineal gland cyst*

\begin{tabular}{|c|c|}
\hline Pre-existing diagnosis & With certain diagnosis $\mathrm{n} ;(\%)$ \\
\hline \multicolumn{2}{|l|}{ Nervous system and eye } \\
\hline Epilepsy & $22(27.8)$ \\
\hline Change of psychological state & $15(19)$ \\
\hline Headache & $14(17.7)$ \\
\hline Vertigo & $3(3.8)$ \\
\hline Disorders of consciousness & $3(3.8)$ \\
\hline Optic nerve swelling & $2(2.5)$ \\
\hline Syncopa & $2(2.5)$ \\
\hline Developmental delay in children & $2(2.5)$ \\
\hline Minimal neurological dysfunction & $2(2.5)$ \\
\hline Dystonia & $2(2.5)$ \\
\hline Cerebral palsy & $2(2.5)$ \\
\hline Astigmatism & $2(2.5)$ \\
\hline Myopia & $2(2.5)$ \\
\hline Dysarthria & $2(2.5)$ \\
\hline Dyslexia & $2(2.5)$ \\
\hline Dysgraphia & $2(2.5)$ \\
\hline Vision disorder & $2(2.5)$ \\
\hline Paresthesia & $2(2.5)$ \\
\hline \multicolumn{2}{|l|}{ Endocrine system } \\
\hline Obesity & $8(10.1)$ \\
\hline Thyroid struma & $3(3.8)$ \\
\hline Autoimmune thyroiditis & $2(2.5)$ \\
\hline \multicolumn{2}{|l|}{ Immune system } \\
\hline Tendency to atopy & $9(11.4)$ \\
\hline \multicolumn{2}{|l|}{ Locomotor system } \\
\hline Scoliosis & $2(2.5)$ \\
\hline Poor posture & $2(2.5)$ \\
\hline \multicolumn{2}{|l|}{ Cardiovascular system } \\
\hline Mitral regurgitation & $2(2.5)$ \\
\hline
\end{tabular}

*The diagnoses presented in only one patient have not been included in Table 2 (ventricular septal defect, neurofibromatosis, chronic sinusitis, hirsutism, asthma, hyperprolaktinemia spuria, menorrhagia, radiculopathy, marfanoid habitus, inguinal hernia, liver steatosis, thrombophilia, abdominal colics, cavernoma, central diabetes insipidus, neurogenic bladder, arterial hypertension, peroneal nerve palsy, Langerhans histiocytosis, congenital glaucoma, Ehlers Danlos syndrome, vesicoureteral reflux, posterior urethral valve, varicocele, hypothyreosis). 
tivity disorder and pervasive developmental disorder (Table 2).

We kept a record of the number of medical examinations undertaken before diagnosis of the pineal gland cyst. The number of medical examinations varied from 2 to 18 , but the median was 10 tests. Ten children were hospitalized only for control MRI of a pre-existing pineal gland cyst (Fig. 2).

The largest group of children did not take any medication (44.3\%), whereas $29.1 \%$ of children took one drug in chronic therapy.

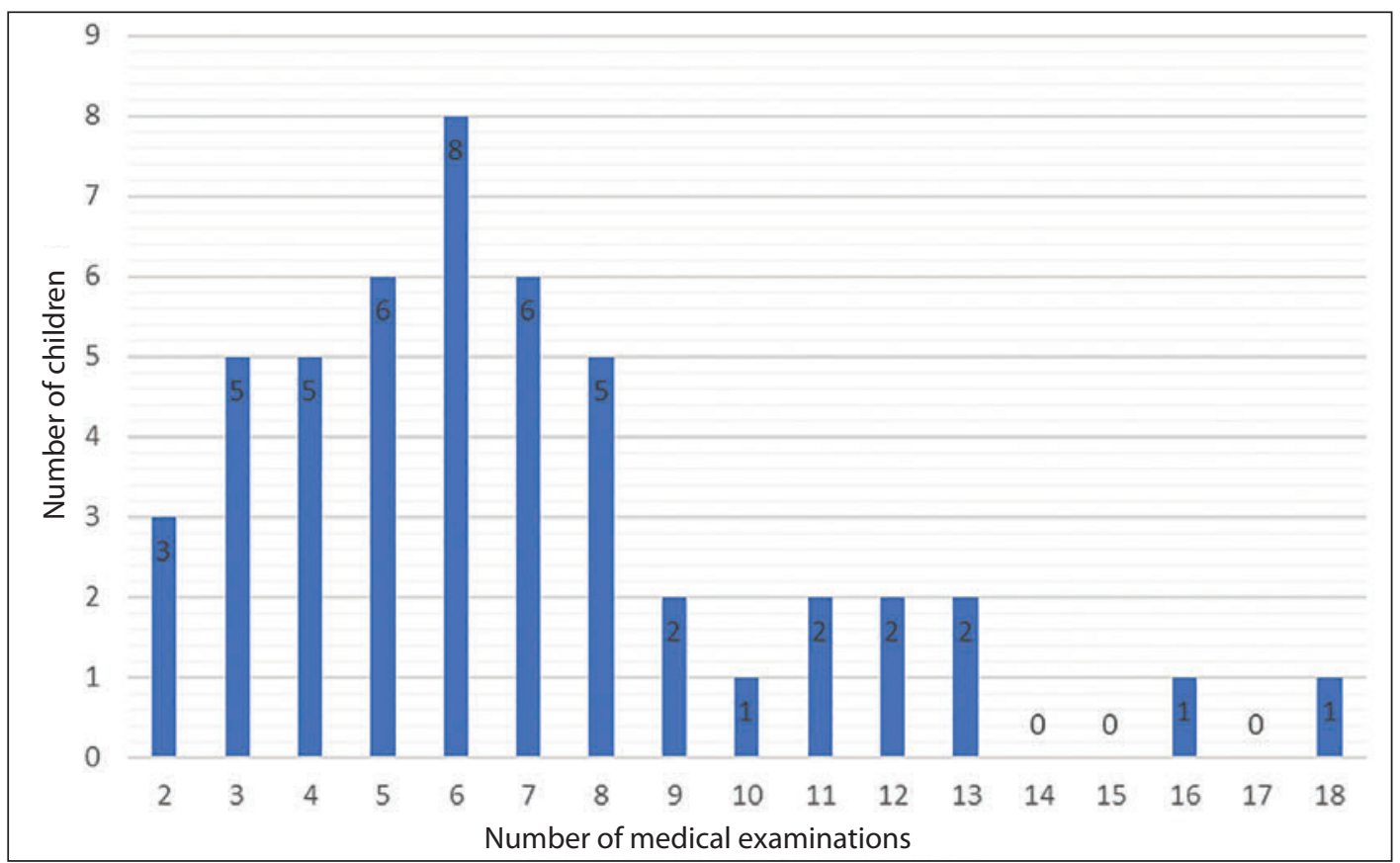

Fig. 2. Number of medical examinations done before diagnosis of a pineal gland cyst.

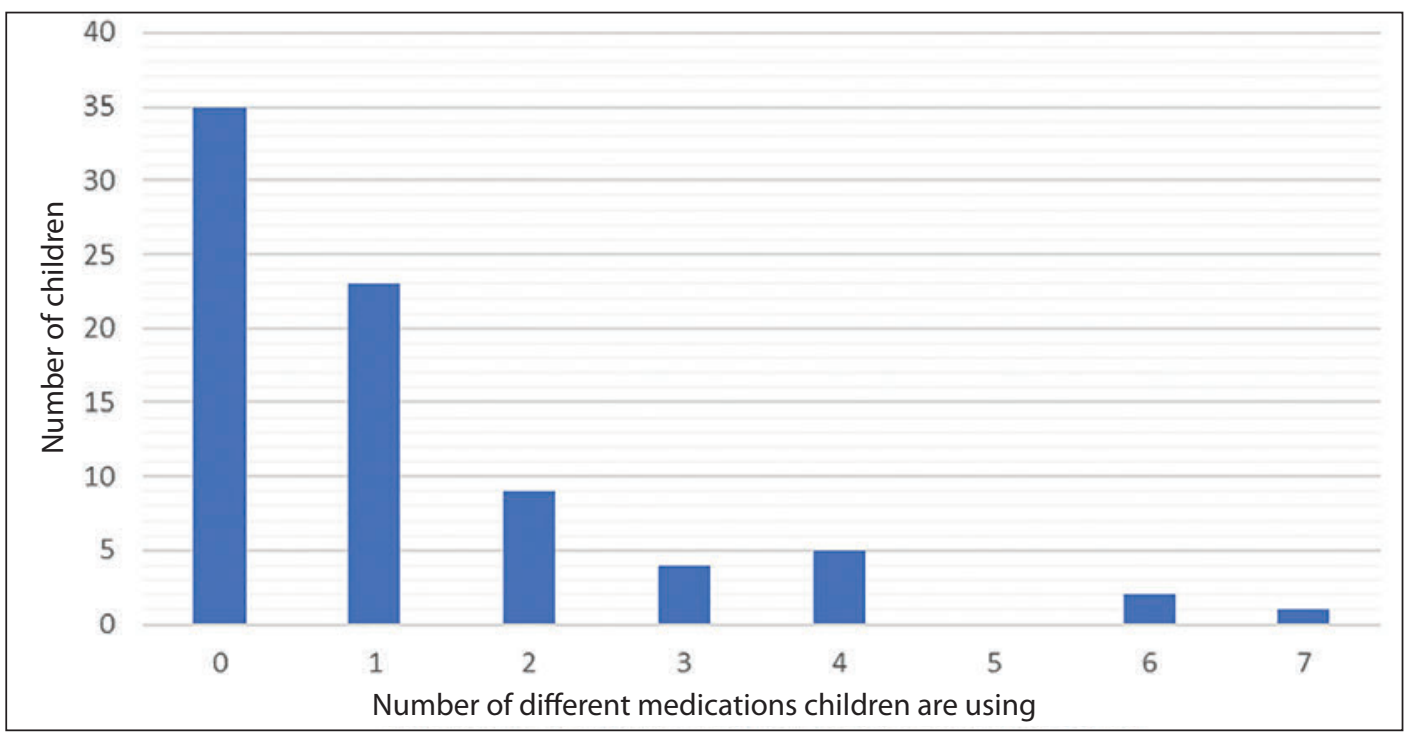

Fig. 3. Distribution of the number of children according to the amount of medications used in their regular therapy. 
The most commonly used drugs were anticonvulsants (27.3\%) (Fig. 3).

No patient had undergone surgery. Out of 79 diagnosed children, 21 children were between 12 and 18 years old and thus satisfied the criteria for psychological testing, conducted by a psychologist. The results of psychological testing were not commented on in particular.

\section{Discussion}

This study was conducted as a retrospective analysis of data collected from the medical documentation of 79 children and adolescents diagnosed with pineal gland cysts. Children were selected from among 30,150 children treated at the Department of Pediatrics at the Clinical Hospital Center, Split in the 10-year period from January 2006 to December 2016. The exact prevalence of the cysts in the children is yet unknown. It is considered that the prevalence of pineal gland cysts in the child population is similar to the one in adults. The largest research conducted on the population of children and young adults included 14,516 patients who underwent magnetic resonance brain imaging, and the prevalence of pineal gland cysts was $2.0 \%$. However, the study involved respondents up to the age of 25 (8).

Although a large amount of data from the 10 -year period has been reviewed, the area gravitating to the Clinical Hospital Center, Split, was not large enough to collect a sufficient number of patients. For this reason, part of the research was explained by descriptive statistics in the form of whole numbers and percentages.

Studies similar to ours have been conducted in Croatia. One study included 84 subjects that all had headache and 38\% had epilepsy (9). The study by Bosnjak et al. shows that the most common symptoms are headache, dizziness, vision problems and obstructive hydrocephalus (5). It is important to emphasize that both studies were conducted on an adult population, as opposed to ours, which is the first study of this type in Croatia performed on patients under the age of 18 . In the 2012 review article by Kahilogullari et al., pineal gland cysts were analyzed in patients under the age of 18 . In this study, headache was mentioned as the most common symptom, while others were loss of consciousness, vision problems and dizziness (10). Unlike all the other studies, we examined not only the symptoms, but also the diagnoses leading to the discovery of the cyst, the size of the cyst, the amount of therapy the children had taken and the amount of medical examinations preformed in order to diagnose the pineal gland cyst.

This study confirms the data observed in adult patients that symptoms such as headache and weaknesses, as well as diagnoses such as epilepsy, might correlate with the existence of pineal gland cysts. The study also shows that most children do not use any regular therapy and if they do, it is usually anticonvulsants. In the absence of any knowledge about the symptoms and accompanying diagnoses in children and adolescents with pineal gland cysts, and bearing in mind that their discovery is usually an incidental finding, the number of medical examinations performed before diagnosing a cyst varied from 2 to 18 . An important finding of this study is that most of our patients had cysts whose size ranged from 0.51 to $1 \mathrm{~cm}(59.5 \%)$. This is the group expected to present with symptoms (5). It would be desirable to carry out the same survey on a larger sample of children, in collaboration with other hospitals in Croatia. This could make medical histories and clinical examinations more important diagnostic procedures leading to a reduction in the number of other diagnostic procedures used, such as imaging. 


\section{Conclusion}

This study confirms that symptoms in children and adolescents, such as headache and weaknesses, as well as epilepsy, might be in correlation with pineal gland cysts. Our conclusions may help to accelerate the process of correlating the diagnosed cysts with the symptoms, as children with nonspecific chronic CNS symptoms, especially epilepsy, are quickly referred to imaging, where the cysts are easily noticed. Due to the rarity of the condition, it would be desirable to repeat the research on a larger number of children.

Authors' contributions: Conception and design: MC and IUS; Acquisition, analysis and interpretation of data: MC and IUS; Drafting the article: IUS; Revising the article critically for intellectual content: MC and IUS; Approved final version of the manuscript: $\mathrm{MC}$ and IUS.

Conflict of interest: The authors declare that they have no conflict of interest.

\section{References}

1. Pineal Gland Anatomy: Overview, Gross Anatomy, Microscopic Anatomy [Internet]. Emedicine.medscape.com. 2017 [cited 2017 Sept 25]. Available from: http://emedicine.medscape.com/ article/1949083-overview\#a1.

2. Møller M, Baeres F. The anatomy and innervation of the mammalian pineal gland. Cell Tissue Res. 2002;309(1):139-50.
3. Sumida M, Barkovich AJ, Newton TH. Development of the Pineal Gland: Measurement with MR. AJNR Am J Neuroradiol. 1996;17(2):233-6.

4. Pu Y, Mahankali S, Hou J, Li J, Lancaster J, Gao J, et al. High Prevalence of Pineal Cysts in Healthy Adults Demonstrated by High-Resolution, Noncontrast Brain MR Imaging. AJNR Am J Neuroradiol. 2007;28(9):1706-09.

5. Bosnjak J, Budisić M, Azman D, Strineka M, Crnjaković M, Demarin V. Pineal gland cysts--an overview. Acta Clin Croat. 2009;48(3):355-8.

6. Al-Holou W, Maher C, Muraszko K, Garton H. The natural history of pineal cysts in children and young adults. J Neurosurg Pediatr. 2010;5(2):1626.

7. Moschovi M, Chrousos GP. Pineal gland masses. Uptodate.com [Internet]. [cited 2017 Sept 25]. Available from: http://www.uptodate.com/contents/pineal-gland-masses

8. Al-Holou W, Garton H, Muraszko K, Ibrahim M, Maher C. Prevalence of pineal cysts in children and young adults. J Neurosurg Pediatr. 2009;4(3):2306.

9. Nankovic S, Hajnsek S, Kovac A, Paladino J, Gadze Z, Sulentic V, et al. Clinical presentation and neurophysiological findings in patients with pineal region expansions — Our 17year experience. J Clin Neurosci. 2013;333:e15.

10. Kahilogullari G, Massimi L, Di Rocco C. Pineal cysts in children: case-based update. Childs Nerv Syst. 2013;29(5):753-60.

11. Jussila MP, Olsén P, Salokorpi N, Suo-Palosaari M. Follow-up of pineal cysts in children: is it necessary? Neuroradiology. 2017;59(12):1265-73. 